\title{
Criminologie
}

\section{Évaluation statique des délits violents chez les délinquants sexuels incarcérés en Belgique francophone}

\section{Thierry H. Pham, Isabelle Debruyne et Audrey Kinappe}

Volume 32, numéro 2, automne 1999

La justice des mineurs

URI : https://id.erudit.org/iderudit/004739ar

DOI : https://doi.org/10.7202/004739ar

Aller au sommaire du numéro

Éditeur(s)

Les Presses de l'Université de Montréal

ISSN

0316-0041 (imprimé)

1492-1367 (numérique)

Découvrir la revue

Citer cet article

Pham, T. H., Debruyne, I. \& Kinappe, A. (1999). Évaluation statique des délits violents chez les délinquants sexuels incarcérés en Belgique francophone.

Criminologie, 32(2), 117-125. https://doi.org/10.7202/004739ar
Résumé de l'article

L'étude compare, à travers des variables statiques, des comportements antisociaux chez trois groupes de délinquants sexuels incarcérés en Belgique francophone. Les trois groupes étaient constitués de (1) 51 violeurs, (2) 46 abuseurs sur mineurs et (3) 41 auteurs d'inceste. Les variables concernaient : (a) le niveau de condamnation ; (b) l'âge relatif aux derniers faits ; (c) le nombre et (d) le type de délits violents et non violents ; (e) le nombre total et (f) le type de victimes. Les résultats suggèrent que par rapport aux deux autres groupes, les violeurs sont plus jeunes et manifestent un nombre plus élevé de délits généraux et violents en particulier. Dans l'ensemble, les résultats sont congruents avec les données de la littérature qui suggèrent que par rapport aux autres catégories de délinquants sexuels, les violeurs manifestent davantage de comportements antisociaux. 


\title{
Évaluation statique des délits violents chez les délinquants sexuels incarcérés en Belgique francophone
}

\author{
Thierry H. Pham ${ }^{1}$ \\ Service psychologique, Prison de Mons \\ et École de criminologie \\ Université catholique de Louvain • Belgique \\ pham@crim.ucl.ac.be
}

Isabelle Debruyne

Service psychologique

Prison de Mons - Belgique

Audrey Kinappe

Service psychologique

Prison de Jamioulx • Belgique

RÉSUMÉ - L'étude compare, à travers des variables statiques, des comportements antisociaux chez trois groupes de délinquants sexuels incarcérés en Belgique francophone. Les trois groupes étaient constitués de (1) 51 violeurs, (2) 46 abuseurs sur mineurs et (3) 41 auteurs d'inceste. Les variables concernaient : (a) le niveau de condamnation ; (b) l'âge relatif aux derniers faits ; (c) le nombre et (d) le type de délits violents et non violents ; (e) le nombre total et (f) le type de victimes. Les résultats suggèrent que par rapport aux deux autres groupes, les violeurs sont plus jeunes et manifestent un nombre plus élevé de délits généraux et violents en particulier. Dans l'ensemble, les résultats sont congruents avec les données de la littérature qui suggèrent que par rapport aux autres catégories de délinquants sexuels, les violeurs manifestent davantage de comportements antisociaux.

1. Les auteurs remercient chaleureusement le Docteur Vandenbroucke, la direction de sept établissements carcéraux, ainsi que la direction du Service des cas individuels du ministère belge de la Justice pour avoir facilité l'accès aux données de l'étude. Sophie Duvivier est remerciée pour son travail relatif au codage des données. 
ABSTRACT - The purpose of the study was to compare static variables related to the antisocial history of three groups of sexual offenders confined in Belgian prisons. The groups were composed by (1) 51 rapists of females, (2) 46 child molesters and (3) 41 incest perpetrators. The variables were: (a) the severity of the sentence pronounced (b) age at the latest incarceration, (c) the number and (d) the type of violent and non violent offences committed (e) the total number and ( $f$ ) the sex of victims. The results showed that rapists were younger than the two other groups. The same subjects exhibited a higher number of both general and violent offences. On the whole, the results are congruent with data suggesting that rapists exhibit more antisocial behaviors in general.

Depuis la fin des années 1950, un important faisceau de données met en doute l'habileté des cliniciens à prédire la dangerosité relative aux comportements violents (voir Shah, 1978 ; Monahan, 1981, 1984 ; Webster, 1990 ; Webster et al., 1994). Afin de remédier à ces lacunes, Monahan, en 1981 déjà, avait préconisé de quantifier les paramètres, tels que l'âge, ainsi que les variables décrivant les comportements criminels passés en vue d'examiner leur validité prédictive au niveau de la récidive.

Dans le domaine de la délinquance sexuelle, la validité prédictive des variables statiques liées aux antécédents délictueux a été soulignée. En effet, Hall (1988) a montré que l'âge, le nombre d'agressions sexuelles sur des personnes adultes, le nombre d'agressions sexuelles sur les enfants et le nombre d'agressions non sexuelles en général étaient plus précis pour l'identification des délinquants sexuels récidivistes que le jugement clinique relatif à la réussite ou à l'échec du sujet à un programme de traitement de type confrontant (variable dynamique). Par ailleurs, les antécédents délictueux, l'âge ou le statut marital prédiraient mieux la récidive sexuelle que les variables dynamiques liées aux évaluations psychométriques relatives à l'anxiété ou à la dépression (Proulx et al., 1997).

Récemment, Hanson et Bussière (1998) ont réalisé une méta-analyse qui couvre 61 études portant sur les évaluations de la récidive sexuelle, la récidive violente non sexuelle ainsi que la récidive générale auprès d'une très large population de délinquants sexuels $(\mathrm{N}=23,393)$. Ces chercheurs soulignent que par rapport aux abuseurs sur enfants, les auteurs de viol présentent des pourcentages nettement plus élevés quant à ces trois catégories de récidive. Parmi les études recensées par Hanson et Bussière, nous pouvons relever de manière illustrative les principaux constats qui suivent. D'une part, à travers des mesures longitudinales effectuées après la période de détention, Hall et Proctor (1987) ont 
relevé que les délits de viol commis sur des adultes permettent de prédire les récidives de violences sexuelles et de violences non sexuelles sur adultes. Par contre, les agressions sexuelles commises sur des enfants laissaient moins bien entrevoir la possibilité de récidive sexuelle vis-àvis de ces derniers. D'autre part, en observant une cinquantaine d'auteurs de viol sur une période de 4 ans, Rice et al. (1990) ont constaté que $59 \%$ de ces sujets ont été arrêtés pour un nouveau délit, $28 \%$ ont à nouveau été arrêtés pour des délits sexuels, $43 \%$ ont été arrêtés pour des délits violents parmi lesquels on trouve de la violence sexuelle. Enfin, en comparant les délinquants sexuels sur mineurs avec les délinquants sexuels sur adultes, Proulx et al. (1997) ont souligné que les auteurs de viol étaient plus jeunes et présentaient un nombre plus élevé de délits antérieurs que les abuseurs sexuels sur enfants.

Les données empiriques qui viennent d'être présentées peuvent donc être mises en parallèle avec les travaux descriptifs, taxinomiques et pronostiques qui suggèrent que parmi les délinquants sexuels, les auteurs de viol se caractérisent par un nombre plus élevé de délits violents (voir Rada, 1978 ; Groth, 1979 ; Malamuth, 1986 ; Lowenstein, 1989 ; Prentky et Knight, 1991). Ce sous-groupe présente donc un intérêt particulier dans la mesure où il se caractérise par des taux élevés de récidive violente et sexuelle (voir Harris et al., 1993 ; Quinsey et al., 1995).

La présente étude comparera un ensemble de critères statiques auprès d'un large échantillon de délinquants sexuels incarcérés en Belgique francophone. Ces sujets sont répartis selon trois groupes : (1) agresseurs sexuels sur femme adulte; (2) agresseurs sexuels sur mineur(e) de moins de 18 ans; (3) agresseurs sexuels intrafamiliaux. Ces trois groupes seront comparés du point de vue des délits commis. La littérature empirique (voir Malamuth, 1986 ; Hall et Proctor, 1987 ; Prentky et Knight, 1991 ; Hanson et Bussière, 1998) permet d'avancer l'hypothèse que par rapport aux deux autres groupes, le groupe de violeurs sur femme adulte manifeste davantage de comportements antisociaux, tant violents que non violents.

\section{Méthode}

Sujets

Les 138 sujets qui font l'objet de cette étude proviennent de 7 prisons belges. Ces sujets ont été arrêtés pour avoir commis une ou plusieurs : (1) agression(s) sexuelle(s) sur femme(s) âgée(s) de 18 ans ou plus (AFM) 
sans lien de parenté ( $\mathrm{N}=51)$; (2) agression(s) sexuelle(s) sur personne(s) mineure(s) âgée(s) donc de moins de 18 ans (AMI) sans lien de parenté $(\mathrm{N}=46)$; (3) agression(s) sexuelle(s) intrafamiliale(s) (AIF) sur un ou plusieurs membres de la famille biologique $(\mathrm{N}=41)$. Dans ce dernier groupe, l'agresseur est le père biologique de la victime. Les délinquants qui ont commis plusieurs types de délits sexuels (inceste et viol sur mineurs extrafamiliaux) n'ont pas été pris en considération. Il en va de même pour ceux qui ont abusé d'adultes de sexe masculin et ceux qui ont manifesté des comportements exhibitionnistes.

\section{Peines prononcées}

Le Tableau 1 indique la répartition des sujets condamnés qui font l'objet d'une mesure d'incarcération. Ces sujets ont été répertoriés selon deux niveaux de condamnation. Les effectifs des trois groupes de délinquants sexuels ne varient pas significativement selon les niveaux de condamnation $\left(\chi^{2}=1.28\right)$. Toutefois, ces données ont été relevées durant les années 1994 et 1995, c'est-à-dire avant l'affaire " Dutroux » qui a défrayé la chronique en Belgique depuis août 1996. Il n'est pas à exclure que les peines pour délits sexuels depuis cette date aient été alourdies.

\section{Variables}

L'ensemble des variables relatives aux délits et aux troubles disciplinaires en détention figurent dans les dossiers personnels appelés dossiers « d'écrou » et « moral » des délinquants. Les variables qui sont décrites dans le Tableau 2 distinguent les délits qui se traduisent par une violence directe (homicide/tentative, coups et blessures) de ceux qui n'impliquent pas une telle violence directe (vol, escroquerie). Pour chaque sujet, les nombres de délits violents et non violents ont été additionnés.

T A B LEA U 1

Durée des peines prononcées

\begin{tabular}{|cccc|}
\hline & $\begin{array}{c}\text { Groupe 1 } \\
\text { Agresseurs sur } \\
\text { femmes majeures } \\
(\text { AFM) } \\
(\mathbf{N}=51)\end{array}$ & $\begin{array}{c}\text { Groupe 2 } \\
\text { Agresseurs sur } \\
\text { mineurs }\end{array}$ & $\begin{array}{c}\text { Groupe 3 } \\
\text { Agresseurs } \\
\text { intrafamiliaux }\end{array}$ \\
$(\mathbf{N}=\mathbf{4 M I})$ & $\begin{array}{c}\text { (AIF) } \\
(\mathbf{N}=\mathbf{4 1})\end{array}$ \\
\hline $0-5$ ans & $30(59 \%)$ & $23(50 \%)$ & $20(49 \%)$ \\
6 ans-perpétuité & $21(41 \%)$ & $23(50 \%)$ & $21(51 \%)$ \\
\hline
\end{tabular}


T A B LEA U 2

Comparaison des variables relatives à l'âge et aux délits violents et non violents

\begin{tabular}{|c|c|c|c|c|c|}
\hline & $\begin{array}{c}\text { Groupe } 1 \\
\text { Agresseurs } \\
\text { sur femmes } \\
\text { majeures } \\
(\hat{A F M}) \\
(\mathrm{N}=51)\end{array}$ & $\begin{array}{c}\text { Groupe } 2 \\
\text { Agresseurs } \\
\text { sur mineurs } \\
\begin{array}{c}(\mathrm{AMI}) \\
(\mathrm{N}=46)\end{array}\end{array}$ & $\begin{array}{c}\text { Groupe } 3 \\
\text { Agresseurs } \\
\text { intrafamiliaux } \\
(\text { AIF }) \\
(\mathrm{N}=41)\end{array}$ & & \\
\hline Variables & $\begin{array}{l}\text { Moyenne } \\
\text { (SD) }\end{array}$ & $\begin{array}{l}\text { Moyenne } \\
\text { (SD) }\end{array}$ & $\begin{array}{l}\text { Moyenne } \\
\text { (SD) }\end{array}$ & $F(2,135)=$ & $p=$ \\
\hline $\begin{array}{l}\text { Âge lors du } \\
\text { dernier délit } \\
\text { sexuel }\end{array}$ & $\begin{array}{l}31,29 \\
(9,44)\end{array}$ & $\begin{array}{c}36,80 \\
(13,10)\end{array}$ & $\begin{array}{l}40,41 \\
(7,71)\end{array}$ & 8,27 & ,0007 \\
\hline $\begin{array}{l}\text { Nombre total } \\
\text { de délits } \\
\text { commis }\end{array}$ & $\begin{array}{c}2,29 \\
(1,03)\end{array}$ & $\begin{array}{c}1,48 \\
(0,86)\end{array}$ & $\begin{array}{c}1,98 \\
(1,17)\end{array}$ & 7,72 &, 001 \\
\hline $\begin{array}{l}\text { Nombre de } \\
\text { délits violents }\end{array}$ & $\begin{array}{c}1,84 \\
(1,34)\end{array}$ & $\begin{array}{c}1,18 \\
(0,94)\end{array}$ & $\begin{array}{c}1,17 \\
(0,74)\end{array}$ & 4,26 & ,016 \\
\hline $\begin{array}{l}\text { Nombre de } \\
\text { délits non } \\
\text { violents }\end{array}$ & $\begin{array}{c}1,23 \\
(1,18)\end{array}$ & $\begin{array}{c}0,78 \\
(0,92)\end{array}$ & $\begin{array}{c}0,80 \\
(1,01)\end{array}$ & 3,72 & 027 \\
\hline Délits violents & $\begin{array}{c}\text { Fréquence } \\
\%\end{array}$ & $\begin{array}{c}\text { Fréquence } \\
\%\end{array}$ & $\begin{array}{c}\text { Fréquence } \\
\%\end{array}$ & $\chi^{2}$ & \\
\hline $\begin{array}{l}\text { Homicide ou } \\
\text { tentative }\end{array}$ & $\begin{array}{c}10,0 \\
(19,6 \%)\end{array}$ & $\begin{array}{c}6 \\
(13,0 \%)\end{array}$ & $\begin{array}{l}1 \\
(2,4 \%)\end{array}$ & 6,24 &, 0434 \\
\hline $\begin{array}{l}\text { Coups et } \\
\text { blessures }\end{array}$ & $\begin{array}{l}34,0 \\
(66,7 \%)\end{array}$ & $\begin{array}{l}19 \\
(41,3 \%)\end{array}$ & $\begin{array}{l}3 \\
(7,3 \%)\end{array}$ & 33,22 &, 00005 \\
\hline $\begin{array}{l}\text { Délits non } \\
\text { violents }\end{array}$ & $\begin{array}{l}\text { Fréquence } \\
\%\end{array}$ & $\begin{array}{c}\text { Fréquence } \\
\%\end{array}$ & $\begin{array}{c}\text { Fréquence } \\
\%\end{array}$ & $\chi^{2}$ & \\
\hline Vol & $\begin{array}{l}23,0 \\
(45,1 \%)\end{array}$ & $\begin{array}{l}10 \\
(21,7 \%)\end{array}$ & $\begin{array}{c}9 \\
(22,0 \%)\end{array}$ & 8,22 &, 0165 \\
\hline Escroquerie & $\begin{array}{c}10,0 \\
(19,6 \%)\end{array}$ & $\begin{array}{c}7 \\
(15,2 \%)\end{array}$ & $\begin{array}{c}5 \\
(12,0 \%)\end{array}$ & 0,959 & NS \\
\hline
\end{tabular}

\section{Procédure}

Entre décembre 1994 et juin 1995, le second auteur de l'article a évalué les dossiers personnels de chaque sujet ayant été condamné pour un ou plusieurs délit(s) sexuel(s). Les variables décrites dans l'étude sont dégagées à partir (1) de la fiche d'incarcération, (2) des exposés des faits, (3) du dossier disciplinaire et (4) du casier judiciaire, (5) de l'extrait de jugement, (6) et de l'expertise mentale lorsqu'elle était disponible au dossier. Une seconde évaluation, entreprise par le troisième auteur de l'article, a porté sur 45 sujets incarcérés. Les accords inter-juges (r-pearson) ont été 
calculés pour chaque variable. La moyenne de ces accords est de .82 et n'est pas inférieure à celle rapportée dans la littérature sur la prédiction actuarielle (voir Rice et Harris, 1995).

\section{Analyse des données}

Les sujets de l'étude proviennent de 7 établissements pénitenciers et ont été intégrés au sein d'un seul et même échantillon. Par ailleurs, les variables décrites au Tableau 2 ont été codées de manière continue. Dans la mesure où la majorité de ces variables comportaient des variances élevées, il a été décidé de réduire l'asymétrie de leur distribution. Ainsi, les données relatives aux délits et aux victimes ont fait l'objet de transformations logarithmiques selon la formule : $\log _{10}(x+1)$. Les trois groupes de sujets ont ensuite été comparés à l'aide d'ANOVA à un facteur. Les différences entre les groupes ont été soumises à des tests post hoc après contrôle préalable de l'homogénéité (test de Bonferroni) ou de la non-homogénéité (test de Dunnett C) des variances.

\section{Résultats}

\section{Variable relative à l'âge}

L'âge lors du dernier délit sexuel differe très significativement entre les groupes. Les tests post hoc révèlent que le groupe AFM est plus jeune que les groupe AMI et AIF $(\mathrm{p}<.01)$.

\section{Variables relatives aux délits}

Le nombre total de délits commis differe très significativement entre les groupes. Les tests post hoc révèlent que le groupe AFM commet plus de délits que les groupes AMI et AIF $(\mathrm{p}<.05)$. Ces mêmes tests révèlent que les agresseurs sur femmes adultes commettent davantage de délits violents que le groupe AIF $(p<.05)$. En effet, un nombre plus significativement élevé de sujets du groupe AFM se sont vus reprocher des délits de tentative d'homicide ou d'homicide et de coups et blessures. Ces résultats sont d'autant plus marquants que la moyenne d'âge du groupe AFM est plus faible que celle des deux autres groupes. En effet, les sujets plus âgés ont théoriquement plus de chances d'avoir commis davantage de délits. Par ailleurs, le nombre total de délits non violents ne differe pas significativement entre les groupes. Cependant, l'interprétation de ce résultat doit être nuancée à la lueur du fait que le groupe AFM est plus 
jeune que les deux autres groupes. Quant aux groupes AMI et AIF, ils ne different pas significativement au niveau du nombre total de délits, ainsi que des délits violents et non violents.

\section{Variables relatives aux victimes}

Le nombre de victimes de sexe masculin differe significativement entre les groupes (Tableau 3). Les tests post boc suggèrent que le groupe AMI a davantage abusé de victimes de sexe masculin que le groupe AIF $(\mathrm{p}<.05)$. Étant donné la spécificité du groupe AFM, le nombre de victimes de sexe féminin varie significativement entre les trois groupes mais les tests post hoc ne différencient pas les groupes AMI et AIF. Les comparaisons deux à deux relatives au nombre total de victimes effectuées à l'aide de la correction liée à la non-homogénéité des variances ne révèlent pas de différence significative entre les trois groupes.

\section{Discussion}

La présente étude constituait une étape introductive à un ensemble de recherches destinées à évaluer les critères diagnostiques qui différencient les groupes de délinquants sexuels incarcérés en Belgique francophone.

Le groupe «agresseur sexuel sur femme adulte » n'encourt pas de condamnations plus lourdes par rapport aux deux autres groupes. Ce

T A B LE A U 3

Variables relatives aux victimes

\begin{tabular}{|c|c|c|c|c|c|}
\hline & $\begin{array}{c}\text { Groupe } 1 \\
\text { Agresseurs } \\
\text { sur } \\
\text { femmes } \\
\text { majeures } \\
(\text { AFM) } \\
(\mathrm{N}=51)\end{array}$ & $\begin{array}{c}\text { Groupe } 2 \\
\text { Agresseurs } \\
\text { sur } \\
\text { mineurs } \\
\\
(\mathrm{AMI}) \\
(\mathrm{N}=46)\end{array}$ & $\begin{array}{c}\text { Groupe } 3 \\
\text { Agresseurs } \\
\text { intrafamiliaux } \\
\\
(\text { AIF }) \\
(\mathrm{N}=41)\end{array}$ & & \\
\hline Variables & $\begin{array}{l}\text { Moyenne } \\
\text { (SD) }\end{array}$ & $\begin{array}{l}\text { Moyenne } \\
\text { (SD) }\end{array}$ & $\begin{array}{l}\text { Moyenne } \\
\text { (SD) }\end{array}$ & $F(2,135)=$ & $p=$ \\
\hline $\begin{array}{l}\text { Nombre total de } \\
\text { victimes }\end{array}$ & $\begin{array}{c}1,82 \\
(1,29)\end{array}$ & $\begin{array}{c}3,41 \\
(4,41)\end{array}$ & $\begin{array}{c}1,56 \\
(0,84)\end{array}$ & 4,22 & ,017 \\
\hline $\begin{array}{l}\text { Nombre de victimes } \\
\text { de sexe masculin }\end{array}$ & $\begin{array}{c}0,06 \\
(0,31)\end{array}$ & $\begin{array}{c}2,15 \\
(4,51)\end{array}$ & $\begin{array}{c}0,12 \\
(0,40)\end{array}$ & 17,06 & ,0005 \\
\hline $\begin{array}{l}\text { Nombre de victimes } \\
\text { de sexe féminin }\end{array}$ & $\begin{array}{c}1,76 \\
(1,33)\end{array}$ & $\begin{array}{c}1,26 \\
(1,40)\end{array}$ & $\begin{array}{c}1,48 \\
(0,85)\end{array}$ & 4,55 & ,012 \\
\hline
\end{tabular}


résultat differe donc des données québécoises rapportées par Earls et al. (1989) qui révélaient que les agresseurs de femmes adultes sont plus lourdement condamnés (98 mois) que les sujets abuseurs d'enfants (68 mois). À ce sujet, des études ultérieures seraient nécessaires en vue de sonder ces critères ainsi que l'incidence de mythes et de croyances relatives au viol auprès des décideurs judiciaires en Belgique francophone.

Les résultats suggèrent que les agresseurs sexuels sur femme adulte se caractérisent par un nombre plus élevé de délits antisociaux en général et de délits violents (homicide/tentative, coups et blessures) en particulier. Ces résultats peuvent être mis en parallèle avec les données de la littérature qui suggèrent que le délit de viol sur femme adulte serait davantage associé à une trajectoire antisociale chronique (voir Quinsey et al., 1995). Ces résultats sont cohérents avec les données méta-analytiques rapportées dans la littérature internationale par Hanson et Bussière (1998) ainsi que celles que Earls et al. (1989) ont mises en évidence chez des délinquants sexuels québécois.

\section{Bibliographie}

Earls, C., Bouchard, L., Aubut, J., Castonguay, L., Laberge, J. et MCKIBBEN, A. 1989. «Étude descriptive des délinquants sexuels », Revue québécoise de psychologie 10 : 30-40.

Groth, A. N. 1979. Men who Rape: The Psychology of the Offender, New York: Plenum Press.

HALL, G. C. 1988. « Criminal Behavior as a Function of Clinical and Actuarial Variables in a Sexual Offender Population », Journal of Consulting and Clinical Psychology 56 : 404-412.

Hall, G. C. et Proctor, W. C. 1987. «Criminological Predictors of Recidivism in a Sexual Offender Population », Journal of Consulting and Clinical Psychology 55 : 111-112.

HANSON, R. K. et BussiÈre, M. T. 1998. «Predicting Relapse : A Meta-analysis of Sexual Offender Recidivism Studies », Journal of Consulting and Clinical Psychology 2 : 348-362.

HARRIS, G. T., RICE, M. E. et QUINSEY, V. L. 1993. «Violent Recidivism of Mentally Disordered Offenders: The Development of a Statistical Prediction Instrument », Criminal Justice and Behavior 20 :315-335.

LOWENSTEIN, L. F. 1989. "Understanding and Treating the Rapist », Criminologist $13: 192-205$.

Malamuth, N. M. 1986. "Predictors of Naturalistic Sexual Agression», Journal of Personality and Social Psychology 5 : 953-962. 
Monahan, J. 1981. Predicting Violent Behavior: An Assessment of Clinical Techniques, Beverly Hills : Sage Library of Social Research.

Monahan, J. 1984. «The Prediction of Violent Behavior : Toward a Second Generation of Theory and Policy », American Journal of Psychiatry 141 : 1015.

PrentKy, R. A. et Knight, R. A. 1991. «Identifying Critical Dimensions of Discriminating Among Rapists », Journal of Consulting and Clinical Psychology 59 : 643-661.

Proulx, J., Pellerin, B., Paradis, Y., McKibben, A., Aubut, J. et Ouimet, M. 1997. "Static and Dynamic Predictors of Recidivism in Sexual Agressors », Sexual Abuse : A Journal of Research and Treatment 9 : 7-27.

Quinsey, V. L., Lalumière, M. L., Rice, M.E. et Harris, G. T. 1995. «Predicting Sexual Violence», p.114-137 in Assessing Dangerousness: Violence by Sexual Offenders, Batterers, and Child Abusers, sous la direction de J.

C. Campbell. Interpersonal Violence : The Practice Series.

QUinsey, V. L., RiCE, M. E. et HARris, G. T. 1995. « The Actuarial Prediction of Sexual Recidivism », Journal of Interpersonal Violence $10:$ 85-105.

RADA, R. T. 1978. Clinical aspects of the rapist, New-York : Grune et Stratton.

RiCE, M. E., et HARris, G. T. 1995. « Violent Recidivism : Assessing Predictive Validity », Journal of Consulting and Clinical Psychology 63 (5) : 737-748.

Rice, M. E., HARris, G. T. et QUINSEY, V. L. 1990. « A Follow-up of Rapists Assessed in a Maximum-Security Psychiatric Facility », Journal of Interpersonal Violence 5 : 435-448.

SHAH, S. A. 1978. «Dangerousness : A Paradigm for Exploring some Issues in Law and Psychology », American Psychologist 33 : 4-237.

WeBster, C. 1990. «Prediction of Dangerousness Polemic », Canadian Journal of Criminology 32 : 191-196.

Webster, C., Harris, M., Rice, M. E., Cormier, C. A. et Quinsey, V. L. 1994. The Violence Prediction Scheme, Toronto : Centre of Criminology, University of Toronto. 